\title{
Vibration Criteria Considered from Case Studies of Active Magnetic Bearing Equipped Rotating Machines*
}

\author{
OSAMI MATSUSHITA ${ }^{\mathrm{a}, \dagger}$, YOUICHI KANEMITSU ${ }^{\mathrm{b}}$, TAKAO AZUMA $^{\mathrm{c}}$ and YASUO FUKUSHIMA ${ }^{\mathrm{d}}$ \\ a Department of Mechanical Engineering, The National Defense Academy, 1-10-20 Hashirimizu, Yokosuka, Kanagawa, Japan 239; \\ ${ }^{1}$ Faculty of Mechanical Engineering, Kyushu University, 6-10-1 Hakozaki, Higashi-ku, Fukuoka, Japan 812-21; \\ 'Ishikawashima Noise Control Ltd., Ryuseido Okubo Bldg. 1-15-18 Hyakunin-machi, Shinjyuku, Tokyo, Japan 169; \\ ${ }^{\mathrm{d}}$ Compressor Design Department, Tsuchiura works, Hitachi Ltd., 603 Kandatsu-machi, Tsuchiurashi, Japan 300
}

(Received 23 April 1998; In final form 6 October 1998)

\begin{abstract}
The main part of turbo machinery is conventionally supported by oil film lubricated bearings. The rotor vibrations can be suppressed within low levels as to satisfy vibration criteria, e.g., ISO standards for general rotors and API 617 for process compressors. Recently, the sophisticated advantages of the active magnetic bearing (AMB) have been increasing the number of applications to industrial rotors. The AMB vibration control design requires the weak support which induces inevitably large vibration amplitude, though it is normal for AMB itself. Vibration criteria indicated by present standards are thus too strict for AMB equipped rotors. The difference of the bearing dynamic characteristics between the oil film lubricated bearing and the AMB compels us to prepare a new ISO standard which recommends the acceptance of higher vibration levels for AMB operation.

In this paper, a case study was executed concerning AMB equipped LP and HP compressors experiencing long-term operation with no major trouble since December, 1992. The rotating rated speed, $10900 \mathrm{rpm}$, is beyond the first bending critical speed with $5300 \mathrm{~kW}$ of power. The average value of the rotor vibration was about $50 \mu \mathrm{mpp}$ in the site operation. By reviewing the rotor design concept and field data of these AMB equipped machines, new vibration criteria are considered for a proposal of ISO standardization.
\end{abstract}

Keywords: Active magnetic bearing, Oil film lubricated bearing, Compressor, Critical speed layout, Vibration evaluation criteria, Flexible rotor, Control

\section{INTRODUCTION}

A few types of active magnetic bearing (AMB) equipped rotating machinery are currently being implemented successfully in the commercial business market since the development phase of the AMB borne rotor was completed. Some successful examples include turbo-molecular pumps, expanders in chemical plants, spindles in machine tools and centrifugal compressors in turbo machinery.

\footnotetext{
* This paper was originally presented at ISROMAC-7.

${ }^{\dagger}$ Correponding author. Tel.: 0468-41-3810, Extn. 2326. Fax: 0468-44-5900. E-mail: osami@cc-nda.ac.jp.
} 
The new concept of AMB is indeed welcome, thanks to its features of being contact free, maintenance free and without mechanical losses.

A typical system supported by the AMB is illustrated in Fig. 1. AMBs are located at both ends of the shaft including adjacent placements of displacement sensors and emergency (auxiliary) ball bearings. The control network for driving the AMB device is shown in Fig. 2. As shown in these figures, each displacement sensor detects the shaft position at bearing portions and its signal is fed back to the compensator. The deviation from the bearing center is put into the PID (Proportional, Integral and Differential actions) controller. The controller drives the power amplifiers to supply the coil current and to generate the magnetic force for the levitation and vibration control. Instead of PID, many modern control laws, e.g., LQ and $\mathrm{H}$-infinity, are being investigated for this servo-feedback control design.

The AMB dynamic characteristics is governed by the controller transfer function. An example of the transfer function of a PID controller is illustrated in the Bode diagram of Fig. 3. The phase lead frequency domain of the phase curve, e.g., $0.1-10$

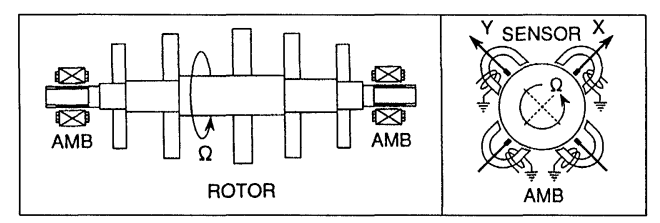

FIGURE 1 Active magnetic bearing equipped rotor system.

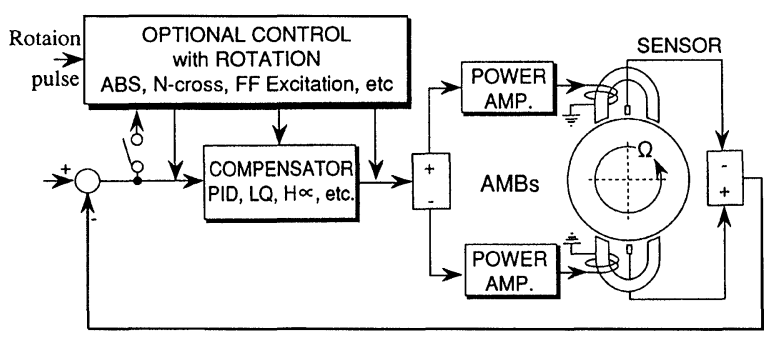

FIGURE 2 AMB control network layout including options: Optional controls work during rotation with inputting the rotational pulse signal. (non-dimension $=$ frequency $/$ the first free-free bending frequency), can provide positive damping to the rotor system, but negative damping in other frequency domains. In this example, the vibrations of the rigid modes and the first bending mode are covered by the positive damping domain.

Compared with conventional oil film lubricated bearings having only positive damping, it can be said that the AMB provides low bearing forces due to limits of the magnetic density. Therefore, concerning bearing support stiffness, the former is strong, and the latter weak. Another opinion might say that the former is too strong, but the latter is preferable.

The main part of turbo machinery is still supported by oil film lubricated bearings. The rotor vibrations can be suppressed within low levels thanks to its stiff support. Vibration evaluations applied to the current type of rotating machines are covered by ISO standards (ISO 7919/1-5) (1996). These ISO standards consequently require low limits for a permissible vibration level. On the other hand, it is hard to suppress AMB rotor vibrations within such low levels due to the feature of its weak bearing stiffness. Furthermore, the AMB design requires the placement of the $\mathrm{AMB}$ at the location of the vibrating portions for effective vibration control. Large vibration magnitude is thus inevitably measured in spite of being normal.

This difference between AMB and oil film bearing dynamics induces a potential conflict

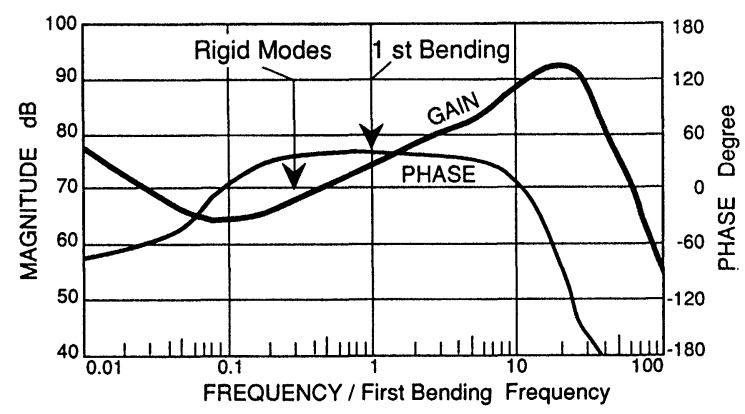

FIGURE 3 AMB controller transfer function: Note that the positive and negative values of the phase curve correspond to the phase lead and lag, i.e., the positive and negative damping factors, respectively. 
between customers and manufacturers. In the case of process compressors equipped with AMBs, a customer requires a manufacturer to comply with the API 617 (1995) regulation which provides for low vibration limits in the same manner as if the compressor were supported by oil film lubricated bearings, though it is actually supported by AMBs. These present limits are too strict for the AMB rotor manufacturing side.

As mentioned above, we need to prepare new ISO standards which will accept the relatively high vibration limits in accordance to the requirement of proper AMB design and operation. This paper proposes new vibration criteria for AMB equipped rotor systems in order to ultimately solve this contradiction.

\section{REQUIREMENTS FOR ISO TC108 ON RELATED VIBRATION REGULATIONS}

A set of ISO standards on rotor vibration regulations for turbo machines is given in ISO 7919 series. ISO 7919: Mechanical vibration of non-reciprocating machines: measurement on rotating shaft and evaluation criteria

Part 1: General guidelines,

Part 2: Large land-based steam turbine generator sets,

Part 3: Coupled industrial machines,

Part 4: Gas turbine sets,

Part 5: Machine sets in hydraulic power generating and pumping plants.

ISO TC108 (Mechanical Vibration and Shock)/SC2 (Measurement and Evaluation of Mechanical Vibration and Shock as Applied to Machines, Vehicle and Structures)/WG1 (Vibration of Machines) has been developing the ISO 7919 series which specifically covers the vibration of turbo machinery; turbines, generators, compressors, pumps, and so on.

When considering centrifugal compressors, these rotors are conventionally supported by oil film bearings, e.g., tilting pad bearings. The AMB has been recently introduced this type of turbo machinery. The consideration of the differences between the bearing dynamic characteristics of both types, i.e., the strong support of the oil film bearing and the weak support of the AMB, are not considered in these series. So far each vibration criteria prepared in ISO and/or API standards govern uniformly oil film bearing equipped turbo machinery. Unfortunately, there are no vibration standards concerning AMB equipped turbo machines.

Because of these concerns, Japanese experts have proposed international standardization for $\mathrm{AMB}$ related technology to the ISO TC108/SC2. The objectives of this standardization are as follows:

(1) to define AMB terminology for promoting mutual understanding for the relevant people,

(2) to resolve conflicts between venders and users with proper designs and operations,

(3) to provide vibration criteria to simplify contract concerns, commissions, etc.,

(4) to promote a design, operation and maintenance guides for AMB equipped rotors,

(5) to accelerate low cost production and widespread applications.

ISO agreed with the necessity of this standardization and organized a new WG7 on Vibration of Active Magnetic Bearing Equipped Rotating Machines. This paper will be contributed for a working draft of WG7 which covers AMB rigid and flexible rotors.

This paper concerns steady state vibrations measured during normal continuous operation, but not resonance vibrations for passing critical speeds. Resonance vibrations are stated in ISO 10814 titled "Mechanical Vibration - Susceptibility and Sensitivity of Machines to Unbalance." According to this standard, rotor resonance vibrations are evaluated by the modal sensitivity, so called $Q$-value. This $Q$-value evaluation was standardized in reference to Balda (1975) and Shiraki and Kanki (1979). 


\section{CASE STUDIES}

\section{Active Magnetic Bearing Applications to Centrifugal Compressor}

Fukushima et al. (1994) showed that AMB equipped centrifugal compressors were manufactured by Hitachi Ltd., Japan, and installed at a refinery plant in Okinawa Sekiyu Seisei Co., Ltd. as the end user under the project contract of Idemitsu Engineering Co., Ltd. and Chiyoda Corporation. As shown in Fig. 4, this turbo machine is a train system which includes a turbine and high and low pressure compressors, noted (HP) and (LP), respectively. These rotors are connected with flexible couplings. These LP and HP compressors are supported by AMBs. This turbine rotor is conventionally supported by oil film bearings. The rotor configurations of the LP and HP compressors are drawn in Fig. 5, including eigen mode shapes obtained by average values of the AMB supporting stiffness. The LP and HP compressors have 7 and 8 stage impellers with the rotor weight of 780 and $930 \mathrm{~kg}$, respectively. As shown in Fig. 5, the control theory demands that the AMB actuator locations should be on such vibrating portion of eigen mode. This demand opposes the desire for low levels of resultant vibrations.
The design specifications for the process compressor is shown in Table I. The rated speed is $10,900 \mathrm{rpm}=182 \mathrm{rps}$ with the nominal shaft power $4120 \mathrm{~kW}$. The specification with respect to the AMB is shown in Table II. These radial AMBs are specified by $L / D=0.98, C / R=6.8 / 1000$ with $C=$ one side clearance $=0.5 \mathrm{~mm}$ per the $\mathrm{AMB}$ journal diameter $=147 \mathrm{~mm}$. It is noted that the clearance of the auxiliary bearing is set by about half the AMB clearance for protecting emergency contacts of the AMB itself.

\section{Comparison Between Oil Film Bearings and AMBs on Critical Speed Map}

One of the most important aspects for designing process compressors is the critical speed map. In order to better understand rotordynamics in comparison with oil film bearings and AMBs, a uniform shaft simplified from actual rotors is selected, having the equivalent diameter of $150 \mathrm{~mm}$ and the same length of the LP as shown in Fig. 5. The critical speed map is thus calculated for the uniform shaft, as shown on a non-dimensional chart of Fig. 6 . The vertical axis is the natural frequency normalized by the first free-free bending mode. The horizontal axis indicates the bearing

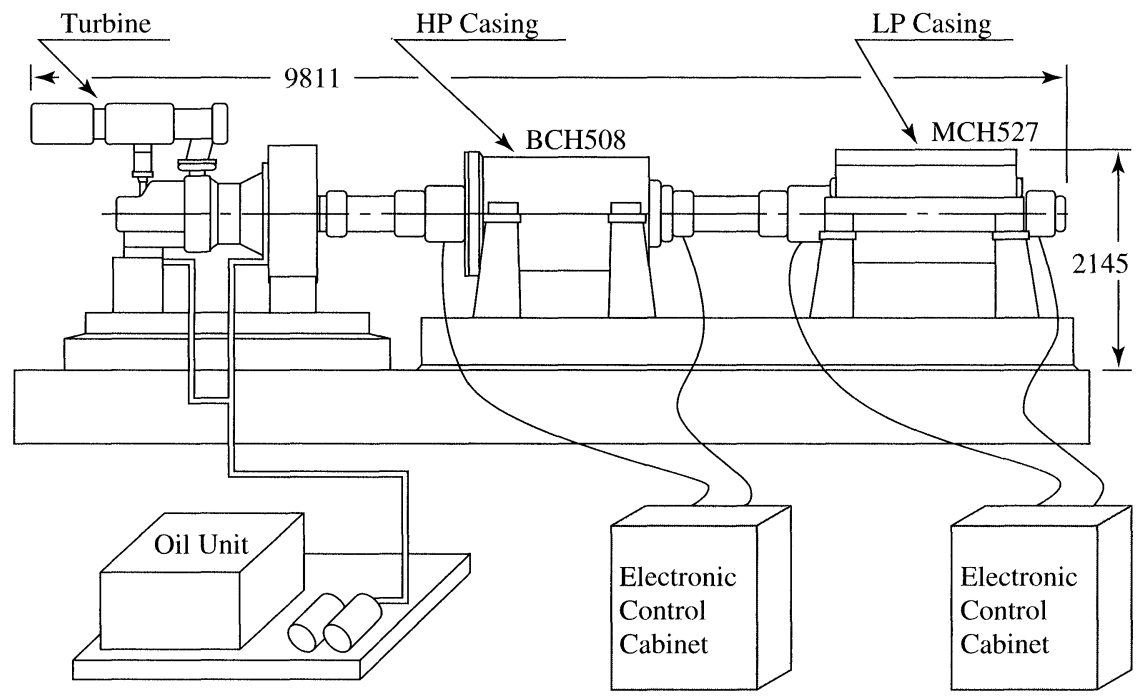

FIGURE 4 Train of AMB equipped compressor. 


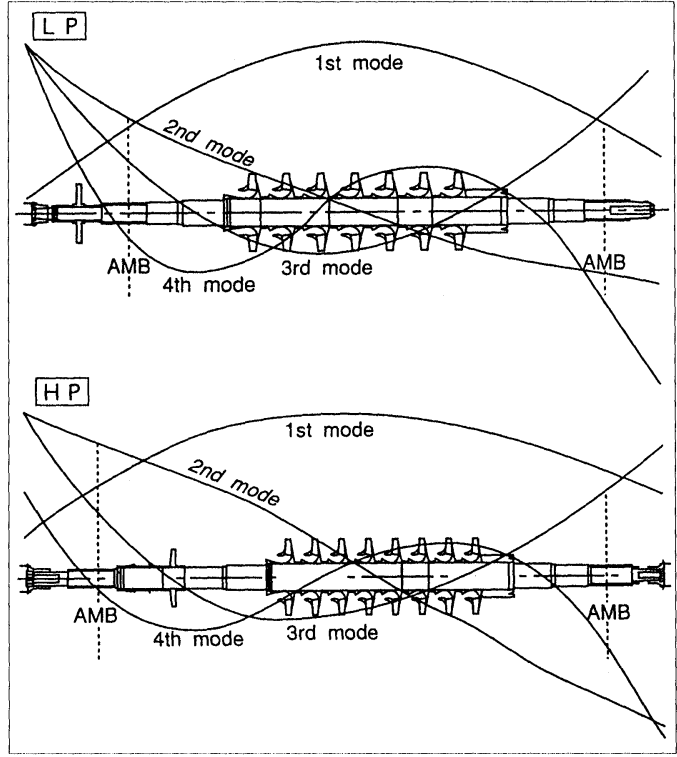

FIGURE 5 Eigen mode shapes of LP and HP rotors: The operational speed is set between the 3 rd and 4 th critical speeds. The AMB locations are avoided to be not node of each eigen mode, because of maintaining enough controllability.

TABLE I Compressor design specification

\begin{tabular}{lcc}
\hline Service & LP casing & HP casing \\
Model & MCH527 & MCH508 \\
Gas & Rich $\mathrm{H}_{2}$ & \\
Flow $\left(\mathrm{N} \mathrm{m}^{3} / \mathrm{h}\right)$ & 43,691 & 43,486 \\
Suction pressure (Mpa) & 0.33 & 0.79 \\
Discharge pressure (Mpa) & 0.91 & 20.3 \\
Rotational speed (1/min) & \multicolumn{2}{c}{$10,900 \mathrm{rpm}$} \\
\multicolumn{2}{c}{$1 \mathrm{rps})$} & \multicolumn{2}{c}{$182 \mathrm{rps}$} \\
Shaft power $(\mathrm{kW})$ & \multicolumn{2}{c}{4,120} \\
Driver rating $(\mathrm{kW})$ & \multicolumn{2}{c}{5,300} \\
\hline
\end{tabular}

TABLE II AMB specification

\begin{tabular}{lcc}
\hline & LP & HP \\
\hline Radial bearing & & \\
Diameter (mm) & 147 & 147 \\
Length $(\mathrm{mm})$ & 150 & 150 \\
Load $(\mathrm{N})$ & 3,822 & 4,557 \\
Capacity $(\mathrm{N})$ & 1,034 & 1,034 \\
Clearance $(\mathrm{mm})$ & 0.5 & 0.5 \\
Radial auxiliary bearing & & \\
Clearance $(\mathrm{mm})$ & 0.23 & 0.23 \\
\hline
\end{tabular}

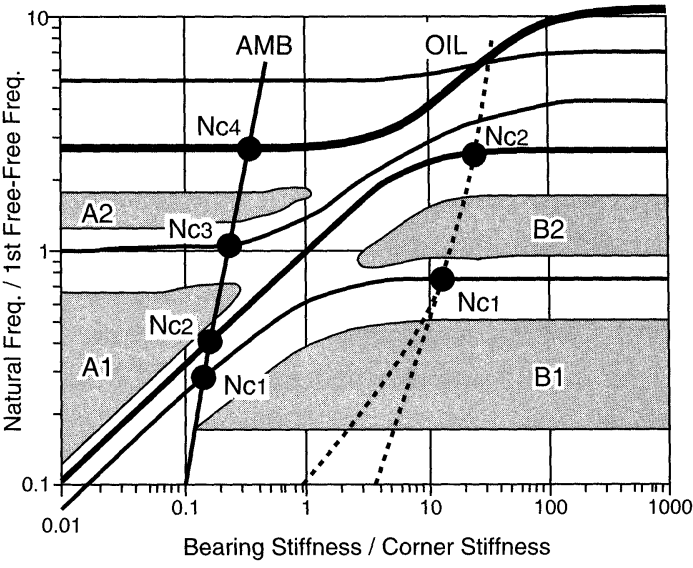

FIGURE 6 A typical critical speed map of a uniform shaft being equivalent to LP rotor: The critical speeds, Nci, are defined by intersections between natural frequency curves and bearing stiffness curves of oil film bearing and AMB. The possible operational speed are set separately from these critical speeds to allow certain margins, as indicated by shaded regions.

stiffness normalized by "corner stiffness" which is identified by a veering point of the first eigen frequency curve. The curve inclines and then holds steady, as the bearing stiffness increases. In other words, the first eigen mode shape changes from the rigid mode to the first pin--pin bending mode. This value will be clear if the map is displayed in the log$\log$ scale.

On the critical speed map, the left and right ends suggest the free-free condition and the pin-pin condition applied to both bearing portions, respectively. The oil film lubricated bearing is generally plotted on the right side due to the strong bearing stiffness. On the other hand, because of weak stiffness, the AMB is plotted on the left side.

In the case of the oil film bearing, its predicted stiffness line (dotted line, $X$ and $Y$ directions) is drawn on the map. The first and second critical speeds are thus marked by the intersections between the eigen frequency curves and the oil film bearing stiffness, noted $\mathrm{Nc1}$ and $\mathrm{Nc2}$, respectively. The compressor to be designed as a rigid rotor must locate the operational speed under the $\mathrm{Nc} 1$ within a certain margin as indicated by the B1 region. For 
the super critical compressor categorized in the flexible rotor, the rotational speed is operated in the range between $\mathrm{Nc} 1$ and $\mathrm{Nc} 2$ within a certain margin as indicated by the $\mathrm{B} 2$ region.

In the case of the AMB, its predicted stiffness is also drawn on the map on the left side, because the stiffness provided by the magnetic force is much weaker compared to the oil film bearing force. Corresponding critical speeds are noted $\mathrm{Nc1}, \mathrm{Nc} 2$, $\mathrm{Nc} 3$ and Nc4. These shaded regions of A1 and A2 become the possible operational regions for so called rigid and flexible rotors, respectively. For instance, turbo-molecular pump, as one of the successful applications of the AMB, is designed to be the rigid rotor so that the rated speed is placed in the $\mathrm{A} 1$ region under the first bending critical speed $\mathrm{Nc3}$. Some AMB equipped compressors are also categorized in this region. The super critical compressors employing the AMB are operated in the $\mathrm{A} 2$ region between the first and second free-free bending critical speeds of $\mathrm{Nc} 3$ and $\mathrm{Nc} 4$. As seen in the Hitachi AMB compressors stated above, this type of super critical AMB compressor is focused to the discussion concerning vibration evaluations.

Generally speaking, the configuration of the oil film bearing is determined by $L / D=1$ and $C / R=$ 0.001 . Since the bearing stiffness is so large, the bearing reaction force is very sensitive to resultant vibrations which must be regulated at low limits. Then, rotors should be well balanced. In the case of $\mathrm{AMB}$, the configuration is mostly seen in about $L /$ $D=1$ and $C=500 \mu \mathrm{m}$. Since the stiffness is so weak, the transmission force from the rotor to the floor, i.e., the bearing reaction force, is still at a low level even if the rotor vibrates at a large level. It can be said that the AMB stiffness requires another new vibration regulation for own usages.

A typical unbalance response curve of $\mathrm{AMB}$ equipped flexible rotors is illustrated in Fig. 7. The unbalance resonance vibration peaks appear at $\mathrm{Nc} 1, \mathrm{Nc} 2, \mathrm{Nc} 3$ and Nc4. The first two modes correspond to the rigid mode critical speeds, i.e., parallel and conical modes, which are commonly well damped by PID controls. The third critical speed Nc3 requires fine tuning to provide a

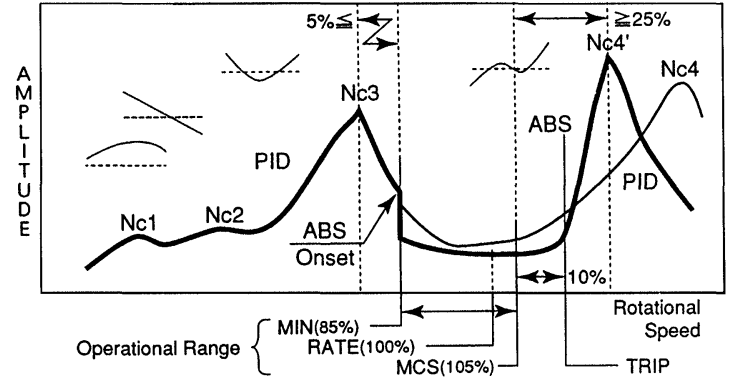

FIGURE 7 A typical unbalance response curve: According to API 617, the separation margins of MIN, MCS and Trip speed are expressed in percent. If the ABS function is switched on at MIN, it works afterward in all of operational speeds.

sufficient damping effect to pass the resonance. The operational speed is thus set in the range between the third ( $\mathrm{Nc} 3)$ and fourth ( $\mathrm{Nc} 4)$ critical speeds with a large margin under the fourth resonance speed. As shown in this unbalance response curve of Fig. 7, the rated speed, the maximum continuous speed (MCS), the trip speed and the minimum speed (MIN) are determined by referring to the API guidelines. It is noted that the operational speed range of the AMB rotor is set between $\mathrm{Nc} 3$ and $\mathrm{Nc} 4$ in the $\mathrm{A} 2$ region and it will be generally more narrow than the $\mathrm{B} 2$ region of the oil film type bearing. The possibility of reduction of the operational speed range for the AMB rotor, compared with the oil film bearing, must be carefully checked in advance.

\section{Critical Speed Layout of AMB Flexible Rotor}

The critical speed map of the LP rotor is shown in Fig. 8 on a linear-linear chart. The AMB dynamic characteristics are featured by the transfer function of the controller. The decomposition of the transfer function can provide the spring $(K)$ and damping forces $(\Omega C)$ depending upon the rotational speed $(\Omega)$, as separately shown in the figure. The former is determined by the real part of the transfer function and the latter is the imaginary part. So called undamped critical speeds are indicated by the intersections between the natural frequency curves 


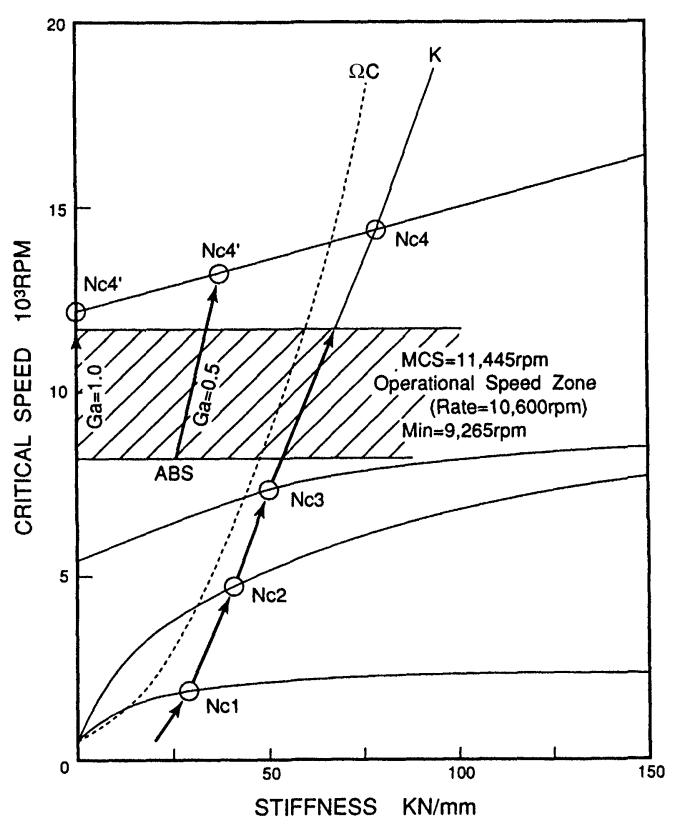

FIGURE 8 Critical speed map of LP rotor: The selection of the ABS gain $\mathrm{Ga}$ in $(0.0-1.0)$ varies the reduction of bearing stiffness.

and the spring force curves as indicated by $\mathrm{Nc1}$, $\mathrm{Nc} 2, \mathrm{Nc} 3$ and Nc4. The operational speed range must maintain enough of a margin against the fourth critical speed Nc4. This reveals a typical example of the critical speed layout for the AMB compressor.

In the case of the AMB, modifications of the transfer function are possible by the implementation of control electronics. For instance, one of the modifications is realized by the so called ABS network innovated by Habermann and Brunet (1985), as shown in Fig. 9. In the figure, the tracking filter including the PLL circuit triggered by the rotational pulse can select only the unbalance vibration which is synchronized with the rotational speed. The substitution in this figure indicates the removal of the filtered unbalance vibration component from the detected displacement signal. The degree of this removal corresponds with the value of this gain $\mathrm{Ga}$. In the case of $\mathrm{Ga}=1$, the absolute removal of the unbalance vibration is achieved. Half the unbalance vibration is removed if $\mathrm{Ga}=0.5$

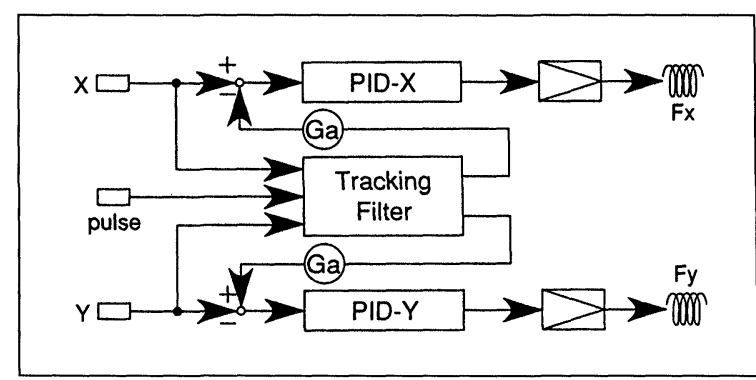

FIGURE 9 Network including ABS: $\mathrm{Ga}=0$ indicates PID only without $\mathrm{ABS} . \mathrm{Ga}=1$ creates no control for unbalance vibrations.

is set. The removed signal is then fed to the PID controller.

Consequently, the AMB stiffness is variable by selecting this gain $\mathrm{Ga}$ so that the corresponding spring curves are drawn on the critical speed map, as shown in Fig. 8.

From the vibration theory point of view, the bearing stiffness for unbalance vibrations is ultimately unnecessary at the rated operation because of no resonance. If ultimately $\mathrm{Ga}=1$ is set, the corresponding fourth critical speed is equal to the second free-free bending frequency as indicated by $\mathrm{Nc}^{\prime}$, instead of $\mathrm{Nc4}$ with $\mathrm{Ga}=0$. It is noted that these downward modifications of AMB stiffness induce the reduction of the fourth critical speed which becomes then closer to the operational speed region. In fact, the unbalance response curve sharply increases toward the $\mathrm{Nc} 4{ }^{\prime}$ critical speed, as shown in the case of ABS $(\mathrm{Ga}=1)$ in Fig. 7. In this way, the rotation under the free-free condition due to the addition of the ABS network becomes dangerous. However, this disadvantage is compensated by the trade-off with several merits: lessening the reaction force transmitted from the rotor to the floor and disappearance of the restriction for dynamic current consumption for vibration control.

\section{Rotation Test Results}

Each LP and HP compressors must independently satisfy the shop performance test in accordance 


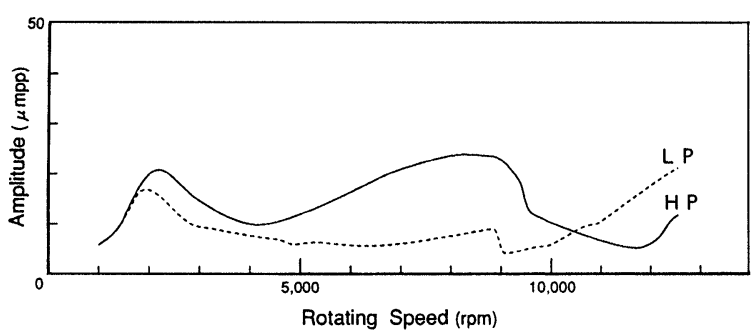

FIGURE 10 Unbalance response curves at mechanical run.

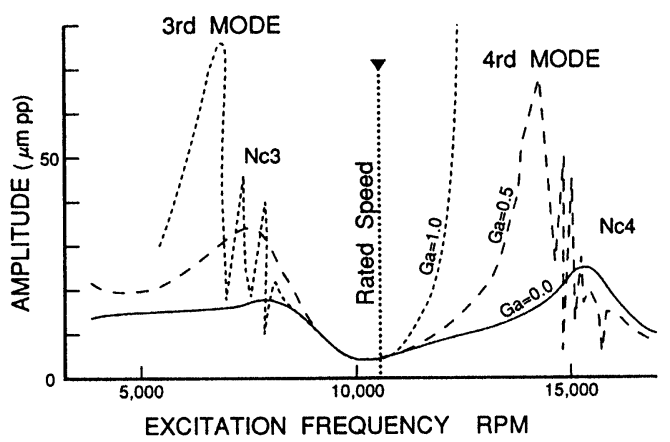

FIGURE 11 Unbalance response influenced by ABS: Note that the ABS induces the reduction of 4 th critical speed and system instability near the 3 rd critical speed.

with ASME PTC-10 and the mechanical run test in accordance with API 617. The test results at the final phase of the mechanical run done at the shop are shown in Fig. 10, as unbalance response curves. Owing to the well rotor balancing, the unbalance vibration amplitude is suppressed enough to the low level of about $20 \mu \mathrm{mpp}$, though the value itself is still larger than the oil film type of compressors.

Depending upon the gain Ga of the ABS circuit shown in Fig. 9, the possible upper limit of the operational speed range is restricted by the unbalance resonance of the critical speed $\mathrm{Nc}^{\prime}$ as illustrated in Fig. 7. This fact is examined through a test by exciting the rotor by harmonic waves at stop. The result is shown in Fig. 11. Instead of a rotational pulse, the excitation harmonic wave signal is concurrent with the trigger signal to the PLL function. It is also noted that the decrease of the $\mathrm{Nc} 4$ critical speed to the $\mathrm{Nc}^{\prime}$ is remarkably recognized by the reduction of the AMB stiffness corresponding upon the selection of the gain $\mathrm{Ga}$.

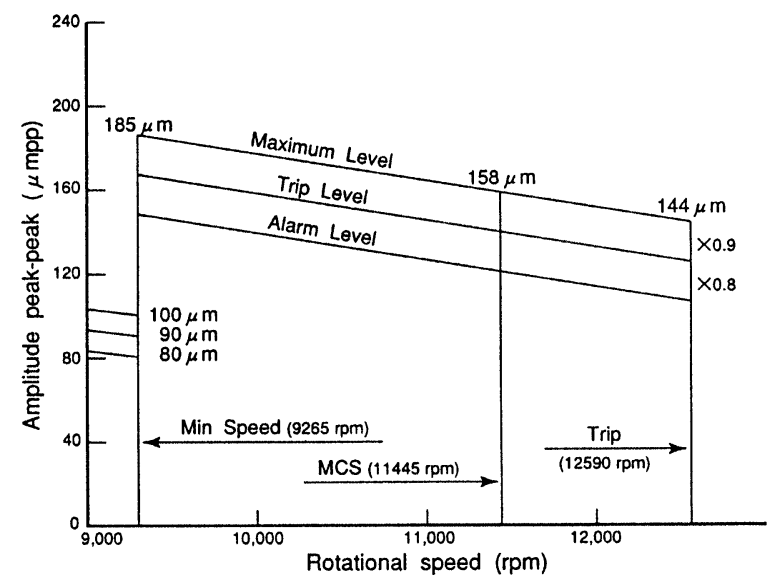

FIGURE 12 Criterion of vibration limitation level (LP).

Vibration limitation levels which should be satisfied were agreed by this project as shown in Fig. 12. This criterion was determined by calculating non-contact conditions between the rotor and stators within a certain margin. The minimum clearance for the non-contact is determined by considering static deflections, vibrational eigen mode shapes and/or rubbing vibration deformations between rotating and stationary parts, e.g., touch down bearing, sensors, labyrinth seal, balancing piston and other parts. The shop test of Fig. 10 proved that these vibration limit levels agreed with the vender and the user are comparably large with conventional oil film bearing type compressors, but are still within normal limits for the AMB rotor.

Since the machine was installed on site for the commission 5 years ago, continuous operations have been completed including the repeat of maintenance every six months without any major problem, as of this writing. An example of field data measured on site is shown in Fig. 13. Steady state data at normal operations concerning the vibration and the current are $50 \mu \mathrm{mpp}$ and $35 \%$ of the current capacity, respectively, as shown in Fig. 13. Both measured values of the vibration and the current are low enough in comparison with each upper limits; about $100 \mu \mathrm{mpp}$ in vibration amplitude and $60 \mathrm{~A}$ in current which are indicated on the vertical axes. An example of vibration magnitude measured during a 


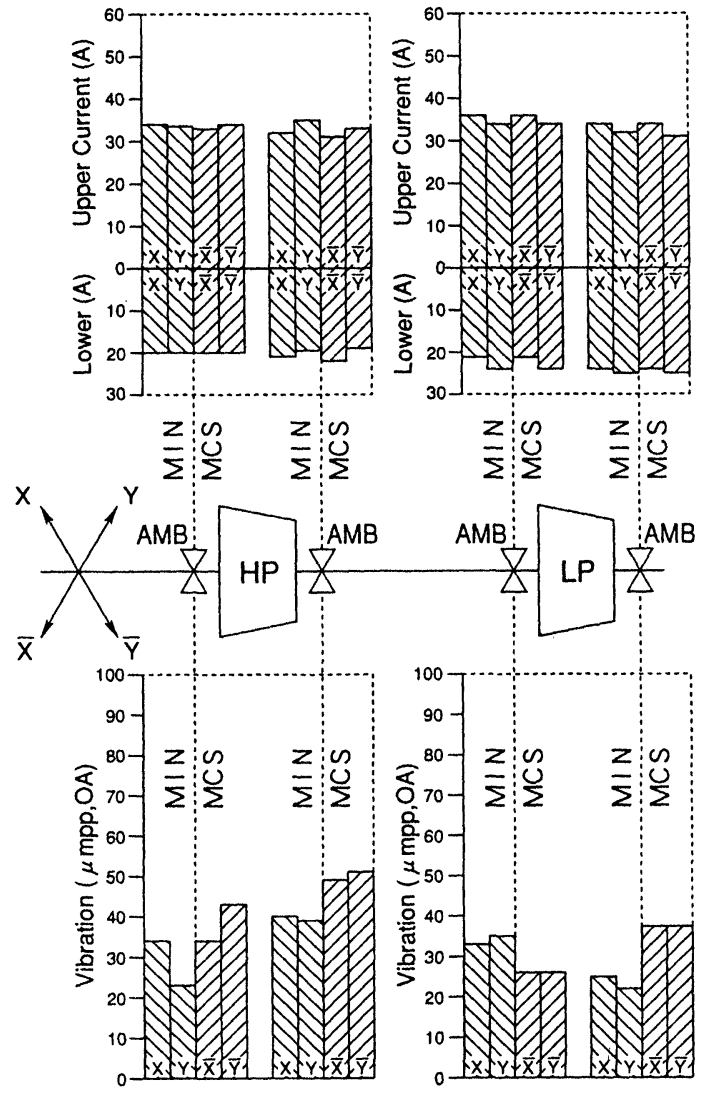

FIGURE 13 Field data (vibration and current): The power amplifiers work as the A-class specification are providing the constant current of $30 \mathrm{~A}$ to upper and lower directional coils. The difference between these upper and lower coil currents is for the rotor levitation.

certain start-stop test is shown in Fig. 14. These vibration data during MCS and MIN operations reveal base line values ranging between 40 and $60 \mu \mathrm{mpp}$. These data fall within predetermined limits.

\section{PROPOSAL DRAFT FOR ISO AMB PROJECT}

\section{ISO TC108/SC2/WG7: AMB Project}

ISO TC108/SC2/WG7 has been organized to discuss $\mathrm{AMB}$ related technology and the required vibration criteria for the AMB equipped rotating machine. The standardization for the AMB project
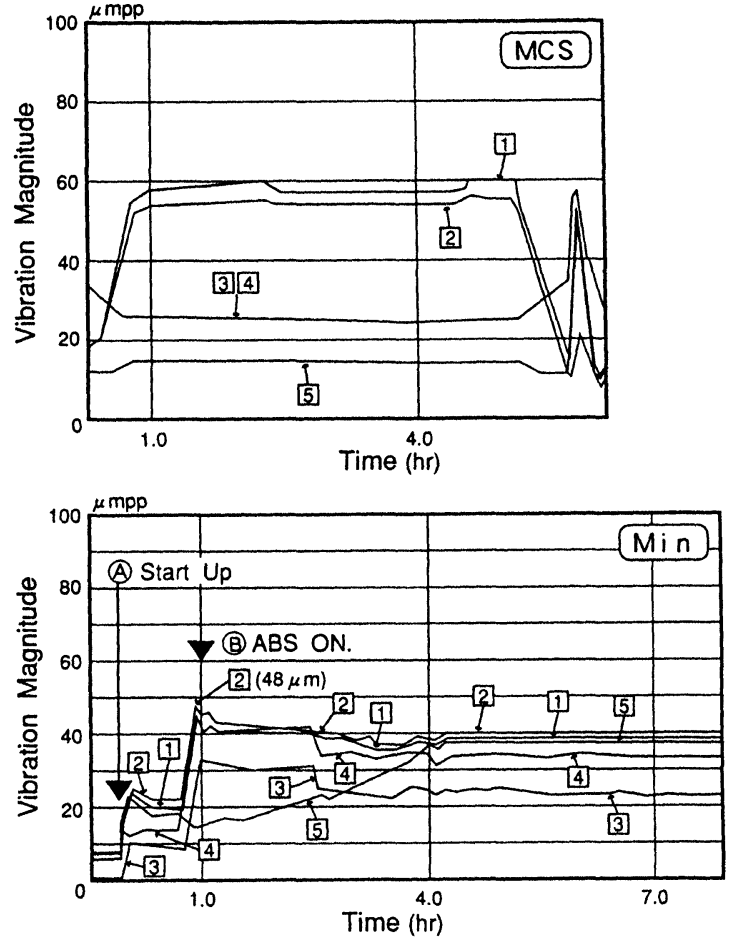

FIGURE 14 Field data (during start--stop): (1)-(4) indicate shaft lateral vibrations and (5) an axial vibration. The upper chart is for LP and the lower for HP.

is just beginning. The initial kick-off meeting of WG7 was held during the program of the ISO TC108 annual meeting at DIN, Berlin, in September, 1997. Every expert from each country can be encouraged to join this project. Kamemitsu et al. (1996) proposed a working draft on AMB vocabulary as a Japanese delegate.

\section{Measurement Procedure}

$\mathrm{AMB}$ equipment in rotating machines has its own displacement sensor for detecting shaft motion within the servo-feedback system. The detected value of shaft vibration by the AMB sensor equipment is subject to these guidelines, but no sensor is additionally needed for this purpose. This rotor vibration evaluation excludes the vibration of the stationary parts of the machine. The AMB coil current should be monitored for this evaluation. 


\section{Criterion 1: Evaluation Zone Limits}

One of the features of the AMB is its large AMB clearance. If the rotor does not make contact with the stators, it can be said that even large vibrations are within normal limits. Therefore, based upon the characteristics of AMB, the criteria concerning the vibrations and current are derived from the following parameters:

(1) No contact with bearings, adjacent displacement sensors, stationary labyrinth seals and auxiliary (emergency, touch down) bearings;

(2) Limit of current compared to power amplifier capacity.

The first parameters concerning each clearance indicate the minimum clearance required for avoiding any rotor rub at any portion of the shafting even in a state of emergency. Thus, the minimum clearance, noted $\mathrm{C}_{\mathrm{min}}$, is commonly defined by the clearance of the auxiliary bearing assuming touch down operations in an emergency. The clearance of the auxiliary bearing is usually determined by half the AMB clearance. The second parameter indicates the current supply capacity, noted Ic, which can provide the maximum current for supplying AMB driving power devices. As an allowable limit for Zone $\mathrm{B}$, it is recommended that the limit values of vibration and current magnitudes be $60 \%$ of each possible maximum value, $\mathrm{C}_{\min }$ and Ic, because these limit values should be a design point when considering excess load and/or unexpected excitation force. The zone table is thus proposed as shown in Table III. The parameters of each zone is described in the guidelines of ISO 7919-1, as follows.

TABLE III Proposed criterion of zone limits

\begin{tabular}{llll}
\hline Zone & \multicolumn{1}{c}{ Vibration } & Current & Evaluation \\
\hline A & $\leq 40 \%$ of $\mathrm{C}_{\text {min }}{ }^{*}$ & $\leq 40 \%$ of Ic & Good \\
B & $\leq 60 \%$ & $\leq 60 \%$ & Accepted \\
C & $\leq 80 \%$ & $\leq 80 \%$ & To be modified \\
D & $>80 \%$ & $>80 \%$ & Not accepted \\
\hline${ }^{*} \mathrm{C}_{\min }=$ minimum clearance and $\mathrm{I}_{\mathrm{c}}=$ current capacity.
\end{tabular}

Zone A: The vibration of newly commissioned machines would normally fall within this zone.

Zone B: Machines with vibration within this zone are normally considered acceptable for unrestricted long-term operation.

Zone C: Machines with vibration within this zone are normally considered unsatisfactory for long-term continuous operation. Generally, the machine may be operated for a limited period in this condition until a suitable opportunity arises for remedial action.

Zone D: Vibration values within this zone are normally considered to be of sufficient severity to cause damage to the machine.

These zone limit values are applied to the measured value of the base line vibration magnitude under steady state operational conditions. According to these criteria, broad-band vibration magnitude less than $100 \mu \mathrm{mpp}$ is thus accepted as Zone $\mathrm{A}$, if $\mathrm{C}_{\min }=250 \mu \mathrm{m}$ of the auxiliary bearing clearance is commonly employed. These values are rather large compared to the present ISO or API 617 criteria, as stated in the following sections.

\section{Criterion 2: Change in Vibration Magnitude}

This criterion provides an assessment of the change in vibration magnitude from a base line. A significant change in broad-band vibration magnitude may occur which would require some actions even though Zone C of Criterion 1 has not been reached. Such changes can be progressive with time or virtually instantaneous and may point to incipient damage or some other irregularity. Criterion 2 is specified on the basis of the change in broad-band vibration magnitude occurring under steady-state operating conditions. When Criterion 2 is applied, it is essential that the vibration measurements being compared are taken at the same sensor location and orientation, and under approximately the same machine operating conditions. Significant changes from the normal vibration magnitudes should be 
regulated to be less than $25 \%$ of the upper boundary value of Zone B, as defined in Table III, because a potentially serious fault may be indicated. When change in vibration magnitude exceeds this value, diagnostic investigations should then be initiated to determine the reason for the change and to decide what further action is necessary.

\section{Comparison with Present Criteria}

Assuming that the minimum clearance of $\mathrm{C}_{\text {min }}=250 \mu \mathrm{m}$ is employed in usual AMB turbo compressors, each zone value of our evaluation criteria mentioned in the previous section is proposed as shown in Table IV. According to our proposal, even in Zone $\mathrm{A}$, the large value of $100 \mu \mathrm{mpp}$ is accepted.

If the present criteria, ISO 7917-3 (Coupled Industrial Machine), concerning the bearing vibrations of large scale turbo machines were applied, the following vibration regulations would be:

Zone A: $\operatorname{Spp}=4800 / \sqrt{N}$ (e.g., Spp $=44 \mu \mathrm{mpp}$, if Trip $=11,445 \mathrm{rpm}$ ),

Zone B: $\operatorname{Spp}=9000 / \sqrt{N}$ (e.g., Spp $=84 \mu \mathrm{mpp}$, if Trip $=11,445 \mathrm{rpm}$ ).

According to the API 617-6 standard criteria regarding centrifugal compressors, the following vibration regulations would be:

$$
\begin{array}{r}
\text { API } 617-6, \mathrm{Lv}=25.4 \sqrt{\frac{12,000}{N_{\mathrm{MCS}}}} \\
\text { and } \mathrm{Lv}<25.4 \mu \mathrm{mpp},
\end{array}
$$

TABLE IV Example of criteria

\begin{tabular}{ll}
\hline Criterion 1: Zone limits & \\
Zone A & Less than $100 \mu \mathrm{mpp}$ \\
Zone B & Less than $150 \mu \mathrm{mpp}$ \\
Zone C & Less than $200 \mu \mathrm{mpp}$ \\
Zone D & Beyond $200 \mu \mathrm{mpp}$ \\
Criterion 2: Vibration change & Less than $38 \mu \mathrm{mpp}$ \\
\hline
\end{tabular}

In the case of $\mathrm{C}_{\min }=250 \mu \mathrm{m}$. where $N_{\text {MCS }}=$ maximum continuous speed (e.g., $\mathrm{Lv}=25.4 \mu \mathrm{mpp}$, if $\mathrm{MCS}=11,445 \mathrm{rpm}$ ). These values are too severe for the commission to exert AMB rotors.

As mentioned in these case studies, these proposed evaluation values seem shockingly large compared to present related criteria. However, these values fall within safe parameters and is our recommendation.

\section{Comparison with Experimental Data}

As stated in the literature, Fukushima et al. (1994), a Hitachi AMB centrifugal compressor, as shown in Fig. 1, was installed in a refinery plant. As shown in Fig. 12 which agreed with this vender-customer project, the alarm level at the maximum continuous speed was determined as $158 \times 0.8=126 \mu \mathrm{mpp}$, i.e., approximately the half of the auxiliary bearing clearance of $230 \mu \mathrm{m}$. The corresponding field data indicate the vibration magnitude of about $60 \mu \mathrm{mpp}$ at maximum which satisfied their own criteria.

On the other hand, because this Hitachi AMB centrifugal compressor employed the auxiliary bearing clearance of $\mathrm{C}_{\min }=230 \mu \mathrm{m}$, our proposed criteria for ISO standards are provided in Table $\mathrm{V}$ in which the limit value of Zone $\mathrm{A}$ is recommended by $\mathrm{C}_{\min } \times 40 \%=92 \mu \mathrm{mpp}$. It is found that this machine has been operating under normal working conditions while satisfying the vibration evaluation criterion of Zone A.

\section{CONCLUSIONS}

Japanese experts have prepared a draft proposal for vibration criterion to be applied to industrial turbo machines equipped with AMBs. Prior to

TABLE V Example of criteria

\begin{tabular}{ll}
\hline Criterion 1: Zone limits & \\
Zone A & Less than $92 \mu \mathrm{mpp}$ \\
Zone B & Less than $138 \mu \mathrm{mpp}$ \\
Zone C & Less than $184 \mu \mathrm{mpp}$ \\
Zone D & Beyond $184 \mu \mathrm{mpp}$ \\
\hline
\end{tabular}

In the case of $\mathrm{C}_{\min }=230 \mu \mathrm{m}$. 
determining the zone limit values, the design review of $\mathrm{AMB}$ equipped compressors was executed with respect to the relationship between $\mathrm{AMB}$ dynamic characteristics and critical speed design of flexible rotors in comparison with the case of oil film bearing type compressor rotors. The vibration limits are proposed through the determination of the minimum possible clearance which avoids all contact and/or rubbing at any location between the rotor and stator sides. The case studies concerning the Hitachi compressors equipped with the AMB are successfully functioning under these criteria.

Since the ISO TC108/SC2/WG7 AMB project was initiated, the committee would like to solicit the review of this draft for vibration evaluation criteria. Your opinion is welcome to help in this endeavor.

\section{References}

API-617/6 (1995) Centrifugal compressors for general refinery service.

Balda, M. (1975) Dynamic properties of turboset rotors, IUTAM Symposium on Dynamics of Rotor, Springer Verlag, pp. $27-55$.

Fukushima, Y. et al. (1994) Totally oil less centrifugal compressor in oil refinery service, Proceeding of Advancement in Bearing and Seal Technologies, Calgary, Canada, pp. 18.1 18.36

Habermann, H. and Brunet, M. (1985) The Active magnetic bearing enables optimum control of machine vibrations, ASME Paper 85-GT-221.

ISO $79191-5$ (1996) Mechanical vibration of non-reciprocating machine (ISO TC108/SC2/WG1).

Kanemitsu, Y. et al. (1996) Japanese proposal for international standardization for active magnetic bearing, Proceeding, of the Fifth International Symposium on Magnetic Bearings, Kanazawa, Japan, pp. 265-270.

Shiraki, K. and Kanki, H. (1979) A new vibration criteria for high speed/large capacity turbo machinery, Proceeding of Eighth Turbomachinery Symposium, pp. 59-70. 


\section{ait \\ ENERGY MATERIALS}

M A N E Y publishing

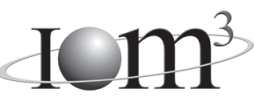

\section{Materials Science \& Engineering for Energy Systems}

Maney Publishing on behalf of the Institute of Materials, Minerals and Mining

The Institute of Materials, Minerals \& Mining

Economic and environmental factors are creating ever greater pressures for the efficient generation, transmission and use of energy. Materials developments are crucial to progress in all these areas: to innovation in design; to extending lifetime and maintenance intervals; and to successful operation in more demanding environments. Drawing together the broad community with interests in these areas, Energy Materials addresses materials needs in future energy generation, transmission, utilisation, conservation and storage. The journal covers thermal generation and gas turbines; renewable power (wind, wave, tidal, hydro, solar and geothermal); fuel cells (low and high temperature); materials issues relevant to biomass and biotechnology; nuclear power generation (fission and fusion); hydrogen generation and storage in the context of the 'hydrogen economy'; and the transmission and storage of the energy produced.

As well as publishing high-quality peer-reviewed research, Energy Materials promotes discussion of issues common to all sectors, through commissioned reviews and commentaries. The journal includes coverage of energy economics and policy, and broader social issues, since the political and legislative context influence research and investment decisions.

\section{CALL FOR PAPERS}

Contributions to the journal should be submitted online at http://ema.edmgr.com

To view the Notes for Contributors please visit: www.maney.co.uk/journals/notes/ema

Upon publication in 2006, this journal will be available via the Ingenta Connect journals service. To view free sample content online visit: www.ingentaconnect.com/content/maney

For further information please contact:

Maney Publishing UK

Tel: +44 (0)113 2497481 Fax: +44 (0)1132486983 Email: subscriptions@maney.co.uk

or

Maney Publishing North America

Tel (toll free): 8662975154 Fax: 6173546875 Email: maney@maneyusa.com

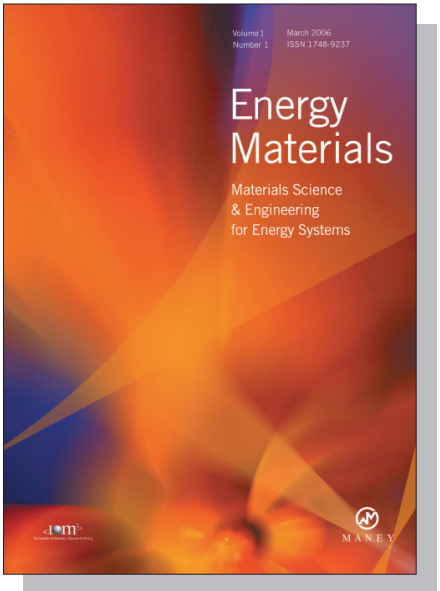

EDITORS

Dr Fujio Abe

NIMS, Japan

Dr John Hald, IPL-MPT, Technical University of Denmark, Denmark

Dr R Viswanathan, EPRI, USA

\section{SUBSCRIPTION INFORMATION}

Volume 1 (2006), 4 issues per year

Print ISSN: 1748-9237 Online ISSN: 1748-9245

Individual rate: $£ 76.00 / U S \$ 141.00$

Institutional rate: $£ 235.00 /$ US $\$ 435.00$

Online-only institutional rate: $£ 199.00 / U S \$ 367.00$

For special $\mathrm{IOM}^{3}$ member rates please email

subscriptions@maney.co.uk

\section{For further information or to subscribe online please visit www.maney.co.uk}



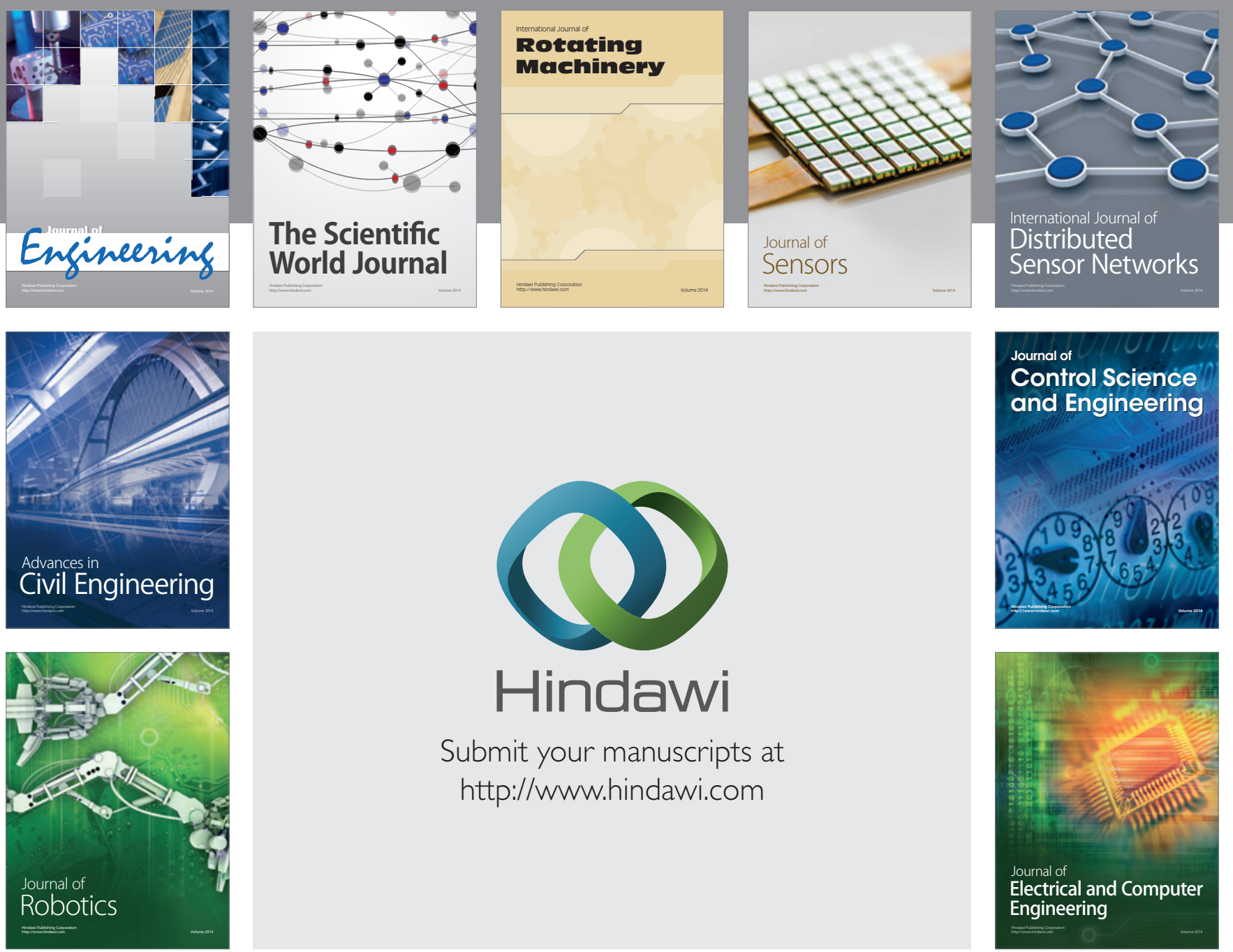

Submit your manuscripts at

http://www.hindawi.com
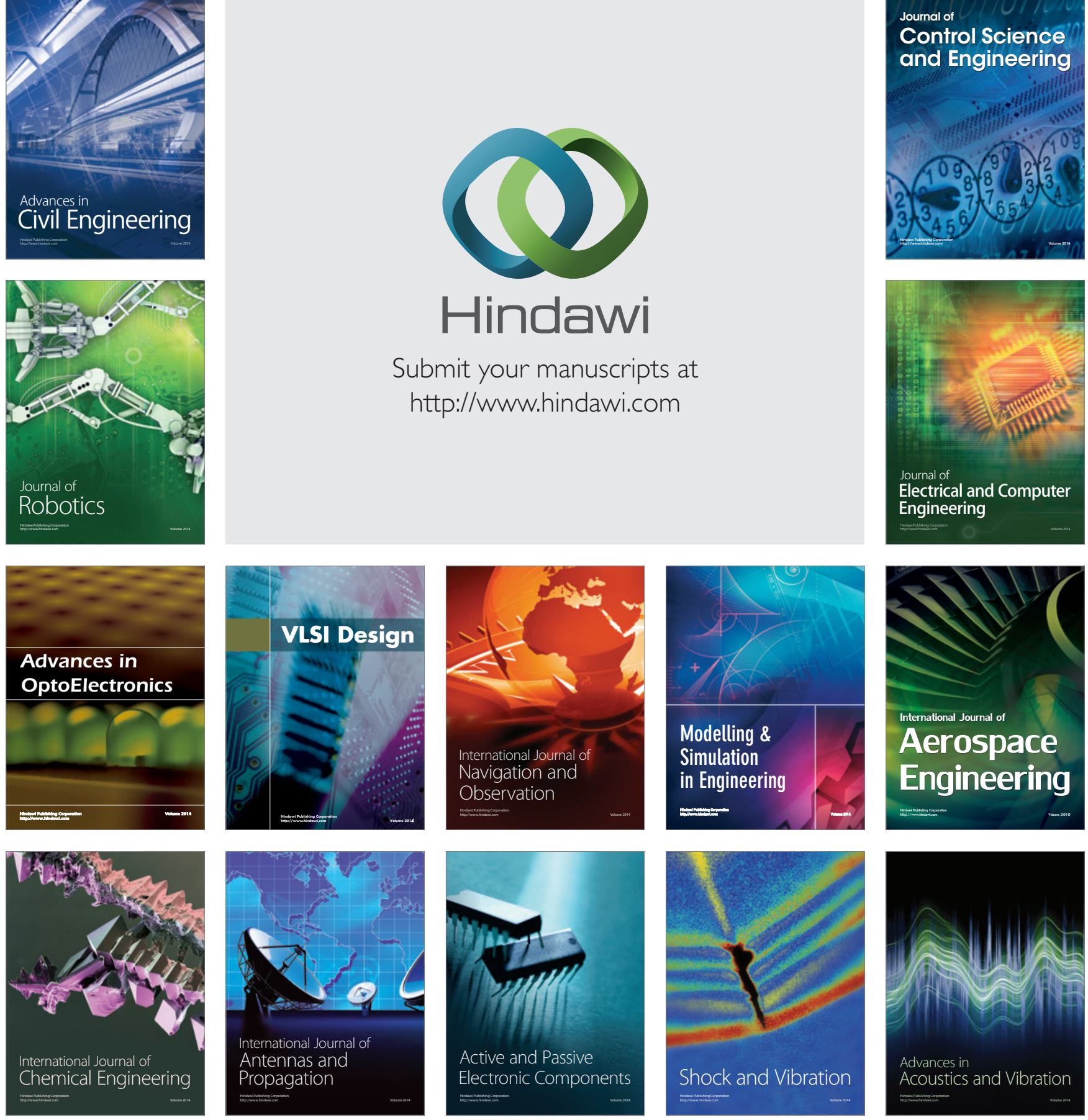